\title{
Dermatological manifestations of measles infection in hospitalised paediatric patients observed in the $2009-2011$ Western Cape epidemic
}

\author{
Bianca Tod, Henri Carrara, Michael Levin, Gail Todd
}

Introduction. Measles is an acute vaccine-preventable infection common in childhood. In this study, the common dermatological signs of measles were designated the 'classic dermatological measles syndrome'.

Methods. We attempted to ascertain the prevalence of 'nonclassic' dermatological measles presentation in 69 paediatric patients admitted to New Somerset Hospital, Western Cape, during the recent South African measles outbreak. The patients were examined and photographed, after informed consent had been obtained, and findings were assessed by 1 dermatology consultant and 6 dermatology registrars. Measles infection was confirmed in 38 of the patients by means of IgM testing. The data were analysed using Stata version 11.1 statistical software.

Outcomes. Of the group, $17.4 \%$ (95\% confidence interval (CI) $8.2-26.6 \%$ ) displayed a 'classic' measles dermatological picture, although all had been clinically diagnosed and admitted as complicated measles cases. Of those serologically confirmed to have measles ( $N=38$ ), $26.3 \%$ (95\% CI 11.6 - 40.9\%) conformed to the 'classic' dermatological picture. Therefore, a significant majority of these patients presented with what was considered in this study to be a 'non-classic' dermatological picture.

Conclusions. Measles infection in a paediatric population requiring admission may frequently present without a full-house 'classic' dermatological picture. Recognised signs in isolation may be of greater value than the classically described syndrome as a whole. 'Non-classic' dermatological forms may occur more frequently than anticipated in complicated cases needing admission. Skin necrosis may be associated with measles.

S Afr Med J 2012;102(6):356-359.
Measles is an acute vaccine-preventable infection common in childhood and caused by a virus of the Paramyxoviridae family. Reports of 'non-classic' measles syndromes ${ }^{1-5}$ include those in the setting of HIV co-infection. ${ }^{2}$ Generally, typical infections are characterised by acute onset of fever, cough, coryza and conjunctivitis; diarrhoea and croup are often present, and secondary bacterial infections such as pneumonia are frequent. Koplik's spots may be noted in the prodromal phase. In the classic form, the rash appears at the hairline and behind the ears on the third or fourth day of illness and then progresses inferiorly. The rash includes erythematous macules on the face with a morbilliform component involving the rest of the body. ${ }^{6}$ For this study, the following were designated the 'classic dermatological measles syndrome': Koplik's spots, other typical oral manifestations (stomatitis), conjunctivitis, and a morbilliform rash with cephalocaudal spread.

An atypical measles syndrome has been reported in patients who were immunised with inactivated measles vaccine and then infected with wild-type measles virus. This type is said to present with a hypersensitivity polyserositis and a rash which may be maculopapular or petechial. In some cases, the rash has been reported as urticarial,

Division of Dermatology, Groote Schuur Hospital and University of Cape Town Bianca Tod, MB BCh

Gail Todd, BSc Agric, MB ChB, FFDerm, PhD

Analytical epidemiologist/biostatistician, Faculty of Health Sciences, University of Cape Town

Henri Carrara, MPH

Division of Allergy, Department of Paediatrics and Child Health, Red Cross War Memorial Children's Hospital, and University of Cape Town

Michael Levin, MB ChB, FCPaed (SA), MMed (Paed), Dip Allerg (SA), PhD purpuric or even vesicular. In patients who received post-exposure prophylaxis in the form of immunoglobulin and in young infants who retained a level of maternal antibody, a modified form of measles has been reported. These patients have a mild prodrome and a mild, shortlasting rash. ${ }^{7}$ The literature reports atypical measles syndromes that may present with diverse skin manifestations, e.g. congested macules and papules starting on the distal extremities and the back. ${ }^{1}$ In the case of human immunodeficiency virus (HIV) co-infection, children may present either without rash, or with an uncharacteristic rash. ${ }^{2}$

Infection rates of measles have declined significantly since the introduction of mass vaccination. ${ }^{6}$ Intermittent epidemics occur when the number of susceptible hosts reaches a critical mass, as was recently observed in South Africa, with 18396 IgM-confirmed cases recorded between January 2009 and February 2011 (most cases being recorded between August 2009 and August 2010) (Fig. 1). ${ }^{8}$ Reasons for such a large epidemic include incomplete or ineffective immunisation (e.g. because of loss of the cold chain), waning immunity in older individuals (particularly those with HIV infection), and incomplete acquisition of vaccine immunity by children in the setting of HIV (reduced amount and quality of immunoglobulin). ${ }^{9-13}$ Infants of HIV-infected mothers inherit less innate antibody protection from measles. ${ }^{11}$ Clustering of non-immune individuals may also contribute to the progress of an epidemic. ${ }^{14} \mathrm{HIV}$-infected children with measles may present with an altered clinical picture. ${ }^{2}$

New Somerset Hospital was a referral centre for suspected cases of measles in the Western Cape during this epidemic. We reviewed the dermatological presentation of 69 paediatric patients admitted to New Somerset Hospital over a period from May to August 2010, and analysed to what degree the dermatological symptoms of those confirmed as having measles coincided with classic descriptions of the skin signs and symptoms of infection.

\section{Objectives}

We aimed to assess: (i) how frequently a patient with laboratoryconfirmed measles presents with skin signs compatible with 


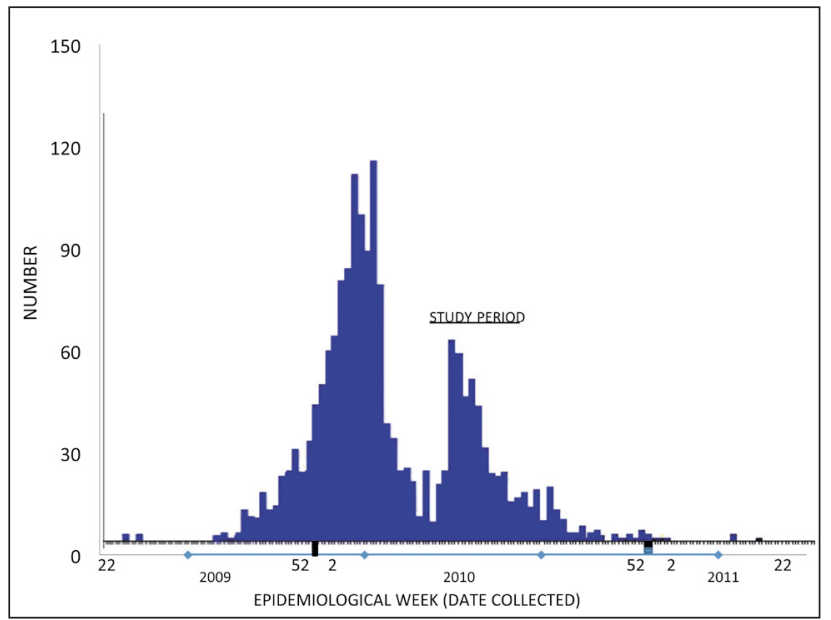

Fig. 1. Positive measles IgM test results: Western Cape, South Africa; June 2009 - 3 June 2011 (adapted from NICD data). ${ }^{7}$

a classic dermatological measles syndrome; (ii) whether HIV influences the dermatological presentation of measles; and (iii) whether malnutrition influences the dermatological presentation of measles.

\section{Methods}

Approval was received from the UCT Ethics Committee (Rec Ref 241/2010). Patients were recruited in the measles-dedicated paediatric wards of Somerset Hospital. Informed consent was taken from the patients' parents. A researcher-administered questionnaire was used to record demographic details and the patient's medical history (including HIV and measles vaccination status). A directed history of the current illness was taken from the primary caregiver. The child was examined and the findings were recorded. Photographs of the patient's dermatological signs were taken. In as many cases as possible, blood tests for measles IgM activity were carried out. The fact that only 41 of the patients were tested reflects changing departmental policy on testing. Serological testing was not appropriate since this was an observational study.

The collected information was assessed by 7 dermatologists consisting of 1 consultant and 6 registrars. With reference to photographs of typical measles skin reactions, they assessed photographs and limited pertinent information regarding the dermatological presentation of each patient obtained at the initial assessment, and determined whether the patient displayed a morbilliform rash or not (Fig. 2). They also rated each patient according to the degree to which they conformed to the 'classic dermatological measles syndrome'. Concordance with the concept of a 'classic dermatological measles syndrome' was judged subjectively and independently by each of the observers. Observers received information regarding the presence of Koplik's spots, conjunctival manifestations, oral manifestations (stomatitis) and cephalocaudal progression of the rash. The observers were blinded to the patients' serological results. They classified the rash that they saw on photographs as morbilliform or not. They rated the patients as scoring between 0 to 10 , with 0 being a patient with no characteristics of 'classic' dermatological measles, and 10 being a textbook case (as described in the introduction of this article). A score of more than 6 out of 10 was taken as a patient profile that could be considered dermatologically typical of measles. The data were analysed using Stata version 11.1 statistical software.

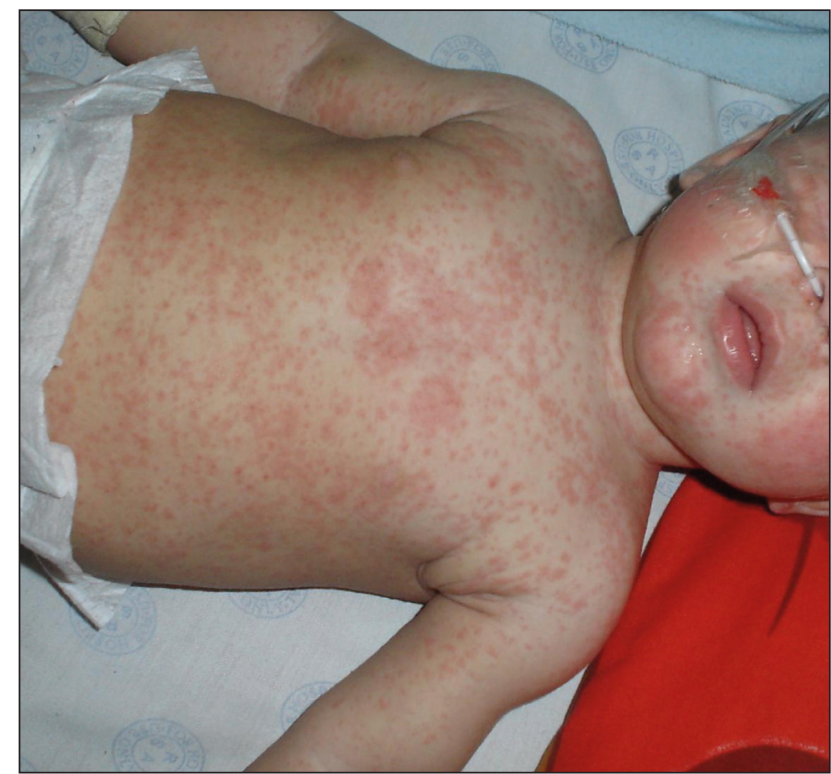

Fig. 2. Morbilliform eruption, part of the 'classic' measles dermatological presentation which included Koplik's spots, other oral manifestations such as stomatitis, conjunctivitis and a history of cephalocaudal spread of the rash.

\section{Outcomes}

In total, 69 patients were assessed - 34 boys (49.3\%) and 35 girls (50.7\%); the median age was 6.0 months (range 2 weeks - 19 months). Forty-one of these patients were tested for measles IgM; 38 (55.1\% of total) of these tested positive for measles infection, 1 patient had an equivocal result, and 2 were negative. Unfortunately, 28 (40.6\%) patients were not tested (Table 1).

Only 1 patient of 59 with a known HIV status was shown to be HIV-positive. HIV status was based on the history given by the patient's guardian (which may or may not have been accurate), the existence of a previous test result on the laboratory system, and any routine testing during admission (if this was done). Ten of the 69 patients had unknown HIV status.

Nine patients were categorised as being malnourished according to the World Health Organization (WHO) classification of nutritional status in children, which uses standard deviation from the norm (or $\mathrm{Z}$-scores) to categorise children. Children whose weight-for-length is between -2 and -3 standard deviations below the norm are classified as moderately wasted, while those less than -3 standard deviations are classified as being severely wasted. Children whose length-for-age is between -2 and -3 standard deviations from the norm are classified as moderately stunted, while those less than -3 standard deviations are classified as severely stunted. The presence of oedema is also noted. ${ }^{9,15}$ Four patients were moderately wasted

Table 1. Demographic data, serological test results and nutritional status

\begin{tabular}{lllll}
\hline Gender & Males & Females & & \\
& $N=34$ & $N=35$ & & \\
Serological test & Positive & Negative & Equivocal & Not tested \\
results & $N=38$ & $N=2$ & $N=1$ & $N=28$ \\
HIV test & Positive & Negative & Unknown & \\
results & $N=1$ & $N=58$ & $N=10$ & \\
Malnourished & Yes & No/not & & \\
& $N=9$ & noted & & \\
& & $N=60$ & & \\
& & &
\end{tabular}


of whom one was oedematous; one was severely wasted; one was severely stunted and severely wasted; one was severely stunted and moderately wasted; one was moderately stunted and moderately wasted. The final patient displayed failure to thrive (moderately underweight for age). Of these patients, 4 had a positive measles test result, 1 was negative, and 4 had no serological tests done. None was rated as having the 'classic' dermatological measles syndrome.

According to the definition of 'classic dermatological measles' (see introduction, methods), only $17.4 \%$ (95\% CI 8.2 - 26.6\%) of the 69 patients (who were admitted as measles cases) conformed to a classic picture (i.e. scored $>6$ ). Of those patients with positive IgM test results for measles, 26.3\% (95\% CI 11.6 - 40.9\%) presented a 'classic' dermatological picture, whereas of those with unknown IgM test results, only 3.6\% (95\% CI 0 - 10.9\%) were rated as having 'classic' dermatological measles (Fig. 3). Therefore most patients presented with 'non-classic' dermatological lesions.

Only $3(7.9 \%)(95 \%$ CI $0-16.9 \%)$ patients with a positive test result were observed to have Koplik's spots. Twelve (31.6\%) (95\% CI $16.1-47.0 \%)$ of the 38 patients with positive test results were noted to have typical oral manifestations. Conjunctival involvement was more common, with $81.6 \%$ (95\% CI 68.7 - 94.5\%) of children with positive test results displaying conjunctival involvement. Cephalocaudal spread of the rash was observed in $76.3 \%$ (95\% CI $62.2-90.5 \%$ ) of patients with positive IgM test results, while $73.7 \%$ (95\% CI 59.0 $88.3 \%$ ) of patients with a confirmed measles test were classified as having a morbilliform rash (Table 2).

Examining combinations of signs, conjunctivitis combined with a rash that had cephalocaudal spread was observed in $60.5 \%$ (95\% CI $44.2-76.8 \%$ ) of patients with positive measles tests. Conjunctivitis with a morbilliform rash was observed in $60.5 \%$ (95\% CI 44.2 -

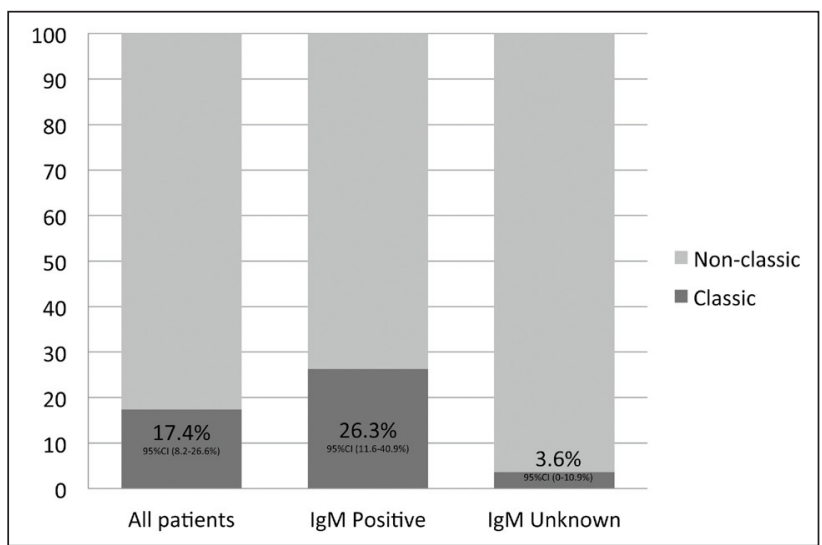

Fig. 3. Percentage of cases with 'classic' dermatological presentation of measles.

\section{Table 2. Frequency of clinical findings}

\begin{tabular}{lccc}
\hline $\begin{array}{l}\text { Signs and } \\
\text { symptoms }\end{array}$ & All subjects (\%) & $\begin{array}{c}\text { Serologically } \\
\text { confirmed (\%) }\end{array}$ & $\begin{array}{c}\text { Not confirmed } \\
(\%)\end{array}$ \\
\hline $\begin{array}{l}\text { Conjunctival } \\
\text { involvement }\end{array}$ & 79.7 & 81.6 & 82.1 \\
$\begin{array}{l}\text { Cephalocaudal } \\
\text { spread }\end{array}$ & 73.9 & 76.3 & 75.0 \\
$\begin{array}{l}\text { Morbilliform } \\
\text { rash }\end{array}$ & 65.2 & 73.7 & 53.6 \\
$\begin{array}{l}\text { Oral } \\
\text { manifestations }\end{array}$ & 36.2 & 31.6 & 39.3 \\
Koplik's spots & 7.3 & 7.9 & 3.6
\end{tabular}

76.8\%). A morbilliform rash with cephalocaudal spread was observed in $52.6 \%$ (95\% CI 36.0 - 69.3\%). Conjunctivitis and a morbilliform rash with cephalocaudal spread were observed in $42.1 \%$ (95\% CI 25.6 - 58.6\%) of patients with positive measles tests.

The dermatological team also noted other skin findings that they observed on examining the photographs, including common skin diseases such as eczema, seborrhoeic dermatitis and nappy rash. These findings did not appear to influence whether the child's dermatological signs of measles were considered to be classic or not. Other isolated presentations such as a rash consisting only of skin-coloured micropapules (in a confirmed case, Fig. 4), and skin necrosis (in $55.5 \%$ of confirmed cases, Fig. 5) were recorded.

The median age of patients assessed (6.0 months) fits in with the fact that most children contracted the illness before immunisation (usually at 9 and 18 months of age). Incomplete immunity from maternal antibodies may alter the response of infants to disease. ${ }^{16}$ That only 2 of the 41 patients who were tested were negative, suggests that the clinical diagnosis of measles was frequently accurate (i.e. the reason for admission). There is a false-negative rate for the

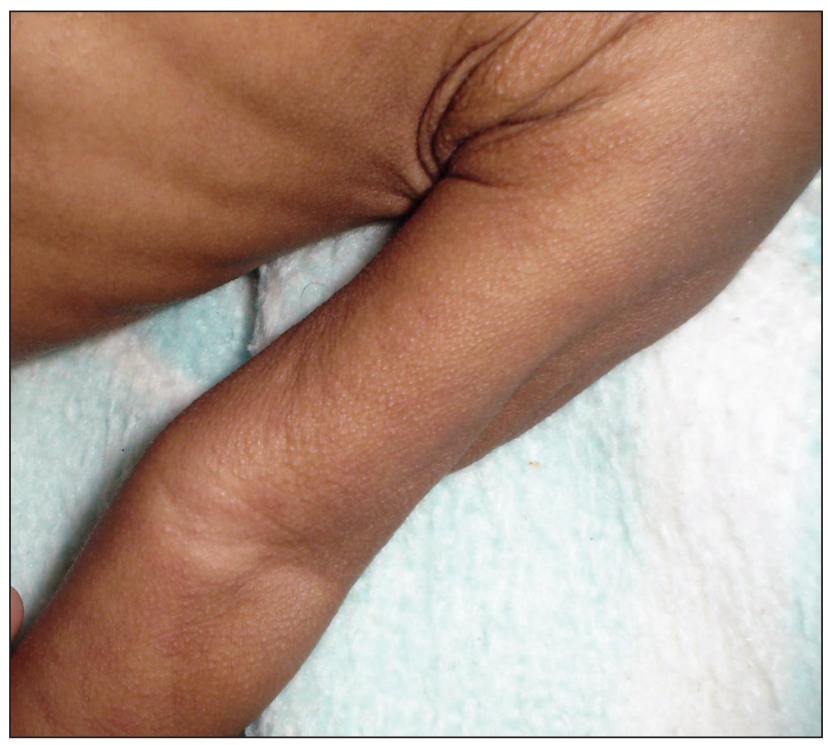

Fig. 4. One study patient with positive measles serology presented with a rash that consisted exclusively of micropapules.

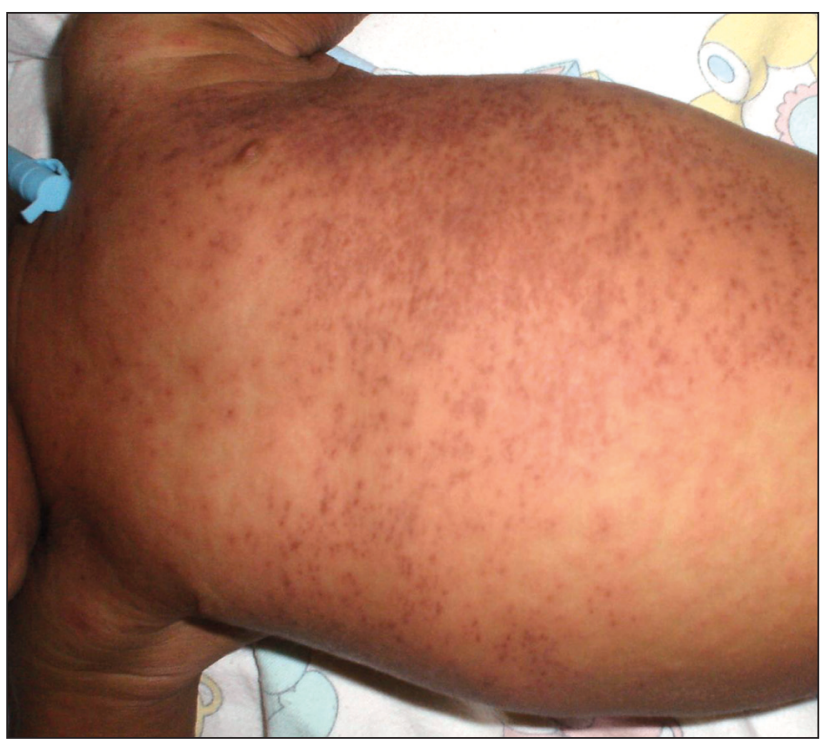

Fig. 5. Study patient with positive serological results displaying skin necrosis. 
measles IgM test, which should be considered. It is important that this population represented patients with illness severe enough to be admitted to hospital, and may not be representative of all cases of measles in the community.

Owing to the low rate of HIV infection in this group, no conclusions could be drawn as to the interaction between the two diseases. The AIDS Committee of the Actuarial Society of South Africa calculate that $1.5 \%$ of children under the age of 2 years in the Western Cape are HIV-positive. ${ }^{17}$ Not all HIV results were known, however.

Because of the small number of patients with malnutrition in the IgM-positive group, no conclusions could be reached in this regard. Of the 9 patients with malnutrition, none displayed a 'classic' dermatological measles picture, and no robust statistical conclusions can be based on such a small number of patients.

In the group as a whole as well as in the IgM-positive group, a low rate of the 'classic dermatological measles' picture was observed ( $17.4 \%$ and $26.3 \%$ respectively). Therefore, the rate of 'non-classic' dermatological presentations was higher than expected. Our study patients who were considered to have 'non-classic' measles presented with dermatological manifestations ranging from what could be considered a modified or mild picture, to a partial picture with only limited expression of the classic signs, to a distinctly unusual presentation (e.g. the child with a rash consisting of micropapules). This may imply that classic dermatological manifestations are not as essential to diagnosing measles as we may have thought. It may also imply that we cling to the presentation of the morbilliform rash combined with other dermatological manifestations too tightly, and only relax this concept in an epidemic, when a susceptible host with any rash or fever is considered to potentially have the disease. Therefore we may frequently miss 'non-classic' dermatological cases in non-epidemic times. The classic signs and symptoms are often present, but not necessarily in the expected combination. The isolated presence of these signs should perhaps be given more weight. It is also important to keep in mind that measles is a disease with multiple systemic symptoms, and considering the dermatological signs and symptoms in isolation represents an oversimplification of its presentation. The low rate of the classic dermatological picture in this study may simply reflect problems with the subjective nature of the study parameters.

In terms of the specific dermatological symptoms reviewed, the low incidence of Koplik's spots may reflect the prodromal timing of their appearance and the difficulty in accurately examining the buccal mucosa of small children. The presence of oral manifestations, such as stomatitis, also had a low predictive value. However, conjunctival involvement was frequently present or noted on history (81.6\%), making this a common clinical sign. The likelihood of the patient having a history of cephalocaudal spread of the rash was high (76.3\%). Morbilliform rash was noted in $73.7 \%$ of positive cases. Interestingly, if this sign stands alone, it may have greater value than the 'classic' dermatological syndrome as a whole. Combinations of two or three signs did not have greater predictive value than the individual signs.

The dermatological team also noted other skin findings that they observed on examination of the photographs. Some observations clearly indicate unrelated co-existent disease (atopic dermatitis, seborrhoeic dermatitis, nappy rash - common in this age group), while others such as skin necrosis (55.5\% of IgM-positive cases) were also observed. One IgM-positive case presented with a rash that consisted only of skin-coloured papules (which may have been consistent with Gianotti-Crosti syndrome). These findings may indicate related skin disease such as drug reactions (acting alone or as co-triggers with viral infection), ${ }^{18-20}$ which may be associated with skin necrosis. They may also be a result of alternative viral exanthems (e.g. Gianotti-Crosti syndrome) or new, as yet unrecorded findings of measles infection. Correlation of such potentially 'new' signs requires greater numbers of patients.

\section{Future directions}

The study was severely hampered by the lack of laboratory confirmation of many patients, which should be improved in future studies. Since only admitted patients were included, there might have been a bias towards children with more severe disease. Communitybased studies should be done in the future. Assessment of potentially 'new' signs of measles, such as skin necrosis, requires greater numbers of patients to make robust conclusions.

\section{Conclusion}

Measles is an acute, vaccine-preventable viral infection. The classic description of this disease focuses largely on its combination of dermatological manifestations (morbilliform rash, conjunctivitis, stomatitis and other oral manifestations such as Koplik's spots). These signs may be individually useful; however, the classic combination may be less common than previously thought, for hospitalised children in particular. This was not peculiar to HIV-infected or malnourished children. We found that in the setting of an epidemic, hospitalised paediatric patients frequently present without this classic combination and we observed a high incidence of skin necrosis. While these patients are diagnosed clinically as having measles in the setting of an epidemic, when one has a high index of suspicion, patients presenting in nonepidemic times without this classic picture may easily be missed.

Acknowledgements. We thank Professor A Westwood and the Department of Paediatrics at New Somerset Hospital, and Drs I Browne, F Esmail, R Kruger, V Lapiner, R Misra and I Steenkamp of the Department of Dermatology, Groote Schuur Hospital.

\section{References}

1. Pang M, Xu JY, Li P, Han XX, Li MY. Clinical analysis of 51 cases of atypical measles syndrome characterized by fever and multiple lung lesions. Zhonghua Jie He He Hu Xi Za Zhi (Chinese journal) 2008:31(10):731-735

2. Permar SR, Griffin DE, Letvin NL. Immune containment and consequences of measles virus infection in healthy and immunocompromised individuals. Clin Vaccine Immunol 2006;13(4): 437-443.

Hall WJ, Hall CB. Atypical measles in adolescents: evaluation of clinical and pulmonary function. Ann Intern Med 1979;90(6):882-886

4. Frey HM, Krugman S. Atypical measles syndrome: unusual hepatic, pulmonary, and immunologic aspects. Am J Med Sci 1981;281(1):51-55.

Tsuruta D, Kobayashi H, Kurokawa I, Ishii M, Takekawa KE. Adult measles with a butterfly rash-like Tsuruta D, Kobayashi H, Kurokawa I, Ishii M,
appearance. Dermatol Online J 2010;16(3):16.

6. Bannister BA, Begg NT, Gillespie SH. Infectious Diseases. 2nd ed. Oxford: Blackwell Science, 2000: 225-229 7. Centers for Disease Control and Prevention. www.cdc.gov/vaccines/pubs/pinkbook/meas.htm (accessed 20 June 2011)

8. National Institute of Communicable Diseases (SA). www.nicd.ac.za (accessed 20 June 2011).

9. Coovadia HM, Wittenberg DF. Paediatrics and Child Health. 5th ed. Cape Town: Oxford, 2004:256-260. 0. De Carvalho V, Marinoni LP, Martins LF, et al. Measles in children with HIV infection: report of five cases. Braz J Infect Dis 2003;7(5):346-352

1. Nilsson A, Chiodi F. Measles outbreak in Africa - is there a link to the HIV-1 epidemic? PLoS Pathog Feb 2011;7(2): e1001241.

12. Nair N, Moss WI, Scott S, et al. HIV-1 infection in Zambian children impairs the development and avidity maturation of measles virus-specific immunoglobulin $\mathrm{G}$ after vaccination and infection. Infect Dis 2009;200(7):1031-1038.

13. Belaunzaran-Zamudio PF, Garcia-Leon ML, Wong-Chew RM, et al. Early loss of measles antibodies Belaunzaran-Zamudio PF, Garcia-Leon ML, Wong-Chew RM, et al. Early loss of measles antibodies
after MMR vaccine among HIV-infected adults receiving HAART. Vaccine 2009;27(50):7059-7064. after MMR vaccine among HIV-infected adults receiving HAART. Vaccine 2009;27(50):7059-7064.
Lowther SA, Curriero FC, Kalish BT, Shields TM, Monze M, Moss WJ. Population immunity to Lowther SA, Curriero FC, Kalish BT, Shields TM, Monze M, Moss WJ. Population immunity to
measles virus and the effect of HIV-1 infection after a mass measles vaccination campaign in Lusaka measles virus and the effect of HIV-1 infection after a mass measles
Zambia: a cross-sectional survey. Lancet 2009;373 (9668):1025-1032.

5. World Health Organization. www.who.int/nutgrowthdb/ (accessed 28 May 2010).

16. Glezen WP. Effect of maternal antibody on the infant immune response. Vaccine 2003;21:3389-3392. 7. Nathea Nicolay, Chair: AIDS Committee, Actuarial Society of South Africa from the ASSA AIDS and Demographic Models.

8. Pinana E, Lei SH, Merino R, et al. DRESS-syndrome on sulfasalazine and naproxen treatment for juvenile idiopathic arthritis and reactivation of human herpesvirus 6 in an 11-year-old Caucasian boyJ Clin Phar Ther 2010; 35: 365-370.

19. Descamps V, Valance A, Edlinger C, et al. Association of HHV6 infection with drug reaction with eosinophilia and systemic symptoms. Arch Dermatol 2001;137(3):301-304.

20. Ichiche M, Kiesch N, De Bels D. DRESS syndrome associated with HHV6 reactivation. Eur J Intern Med 2003;14:489-500.

Accepted 8 February 2012 